\title{
About electrical and mechanical behaviour of low-voltage I\&C cables used in nuclear power plants
}

\author{
Simone V. Suraci*, Davide Fabiani, Stefano Bulzaga \\ Department of Electrical Engineering, University of Bologna, Bologna 40126, Italy
}

Corresponding Author Email: simone.suraci@unibo.it

https://doi.org/10.18280/mmc_c.790303

Received: 10 April 2018

Accepted: 14 May 2018

\section{Keywords:}

low-voltage control cables, postirradiation effect, degradation of polymers, nuclear power plants

\begin{abstract}
This paper focuses on the electrical and mechanical properties of irradiated and thermallystressed NPP cables used for instrumentation and control (I\&C). These cables show some noteworthy changes after few years of uncontrolled environment conditions due to a phenomenon called post-irradiation effect.

Strong post-irradiation effects, e.g. reduction of mechanical properties, raise of crosslinking and oxidation grade, were found on XLPE cable insulation years after irradiation.

These degradation mechanisms can be correlated with electrical measurements in which imaginary permittivity values raise, in particular at low-frequencies where interfacial phenomena take place. This behavior can be linked to the increase of interfacial area between amorphous and crystalline region of polymer, supporting the evidence that postirradiation effects can lead to polymer crosslinking during a storage period even at room temperature.
\end{abstract}

\section{INTRODUCTION}

Low-voltage cables are copiously used in nuclear power plants (NPP) for power transmission, control systems and signal communications. Depending on their application and position in NPPs, cables are exposed to different environmental conditions, e.g. radiations, high temperature and mechanical stresses, particularly inside the containment area. Electric cables are one of the long-life equipment of NPPs, for this reason it is necessary to monitor their behavior throughout the whole lifetime of the considered plant. Indeed, early cable degradation could result in unavailability of important equipment for safety as well as causing dangerous plant transients and shutdowns [1-3].

Since most of the NPPs commissioned in ' 70 s and ' 80 s are now reaching end-of-life and decommissioning costs are very high, electric utility companies are more and more interested to extend the operating time of old NPPs up to 40 more years; to do so, evaluating degradation condition of insulation and predicting residual life of I\&C cables are very critical issues.

Cable insulation is made of polymer-based compounds, whose degradation state is imputed to both radiation and high temperature existing in nuclear plants. Their degradation state is conventionally evaluated through destructive techniques like tensile testing and thermal analysis [3].

In this paper, we focused our attention on electrical diagnostic techniques that are, at the moment, not commonly used for insulation diagnostics, but they are becoming more and more important because of their non-destructiveness [4].

The European Project FP7 ADVANCE (Ageing Diagnostics and Prognostics of low voltage I\&C cables), finished in 2014, focused the study on the ability of electrical condition monitoring (CM) techniques to detect local and global cable ageing as a result of accelerated radiation and thermal aging. Results obtained by electrical CM techniques were compared and correlated to results gained by traditional $\mathrm{CM}$ methods for validation. As an example, modification of electrical permittivity was found to be associated with oxidation induced by aging [5-6].

Unfortunately, accelerated aging brings to diffusion limited oxidation (DLO), which does not permit a homogenous distribution of oxygen inside the material [7]. In addition, material properties change even after several months after irradiation, due to slow diffusion and reaction rate of some chemical radicals generated by aging. This phenomenon is known in literature as post-irradiation effect [8].

The aim of this paper is, on the one hand, to investigate how electrical properties change after some storage period (four years after irradiation in this case) due to the post-irradiation effect; on the other hand, to correlate physical-chemical degradation with non-destructive electric characterization of polymers.

\section{EXPERIMENTAL SETUP}

\subsection{Specimens}

This paper analyzes I\&C Alcatel RG59B cable specimens with XLPE insulation used in nuclear power plants [4]. Morphologically, low-voltage cables are made up of an external sheath, shielding components, primary insulation and conductor.

Outer sheath and shielding components were detached from cables as to focus analysis on primary insulation.

Cable aging was performed using typical NPP environmental conditions, in particular high temperature and radiation.

Specimen characteristics are the following:

(1) Conductor: copper, $\varnothing 0.6 \mathrm{~mm}$; 
(2) Electrical insulation: XLPE, thickness $1.6 \mathrm{~mm}$;

(3) Dose rate: $0.42 \mathrm{kGy} / \mathrm{h}$;

(4) Temperature: $85^{\circ} \mathrm{C}$.

Cables were tested at delivery time and after four years of uncontrolled environment storage, in order to detect the possible post-irradiation effect.

\subsection{Accelerated aging}

The accelerated ageing process was performed at the ROZA facility (ÚJV Řež, Czech Republic). The aging process was fulfilled thanks to a ${ }^{60} \mathrm{Co} \gamma$-ray source with temperature set at $85^{\circ} \mathrm{C}$. Maximum aging time was $1000 \mathrm{~h}$ and cable specimens were removed for analysis every $200 \mathrm{~h} \mathrm{[4].}$

The presence of high dose rates prevents the diffusion of oxygen molecules in the inner part of specimens, so that oxygen groups can only be localized in the outer layers of the irradiated polymers.

This phenomenon is known as DLO [7], as already mentioned in the previous section. DLO affected specimens analyzed in this paper due to the large thickness of cable insulation.

\subsection{Measurements}

A NovoControl Alpha Dielectric Analyzer V2.2 with applied voltage of $3 \mathrm{~V}_{\mathrm{rms}}$ was used to investigate dielectric response of specimens as a function of frequency in the range of $10^{-2}-10^{6} \mathrm{~Hz}$. Input voltage was applied to the central metal conductor, and output signal was measured from a metallic shield placed on the external layer of the insulation.

Mechanical properties of specimens, e.g. elongation-atbreak $(\mathrm{EaB})$, Young modulus and strength at break, were analyzed using a Instron 5566 Universal Testing Machine. This device measures the requested physical quantity by stretching the sample until it breaks while recording load and extension. The chosen extension rate is $50 \mathrm{~mm} \mathrm{~min}^{-1}$, as required by standards [9]. Because of data dispersion, up to six tests have been carried out for each aging time.

\section{EXPERIMENTAL RESULTS}

\subsection{Dielectric spectroscopy results}

Figure 1 and 2 show dielectric spectroscopy results in terms of real $\left(\varepsilon^{\prime}\right)$ and imaginary $\left(\varepsilon^{\prime \prime}\right)$ part of permittivity as a function of frequency for different aging times. It is worth noting that, in most cases, both factors increase with aging time. For this reason, complex permittivity $\varepsilon$ is usually used as a marker for aging grade of polymers [6].

Focusing on the real part of $\varepsilon$, an interesting behavior shows up in the comparison between tests made at delivery time (Fig. 1.a) and after four years of storage (Fig. 1.b). At low frequencies, the real part of permittivity rises during storage, keeping the same variation with aging time before and after storage. On the contrary, in the high frequency range, $\varepsilon$ ' decreases with storage time for every aging level but its variation among aging levels is smaller in after storage tests.

Moreover, Figure 1.b shows a polarization peak in the 10$100 \mathrm{~Hz}$ frequency range, which can be attributed to interfacial phenomena as discussed further on.
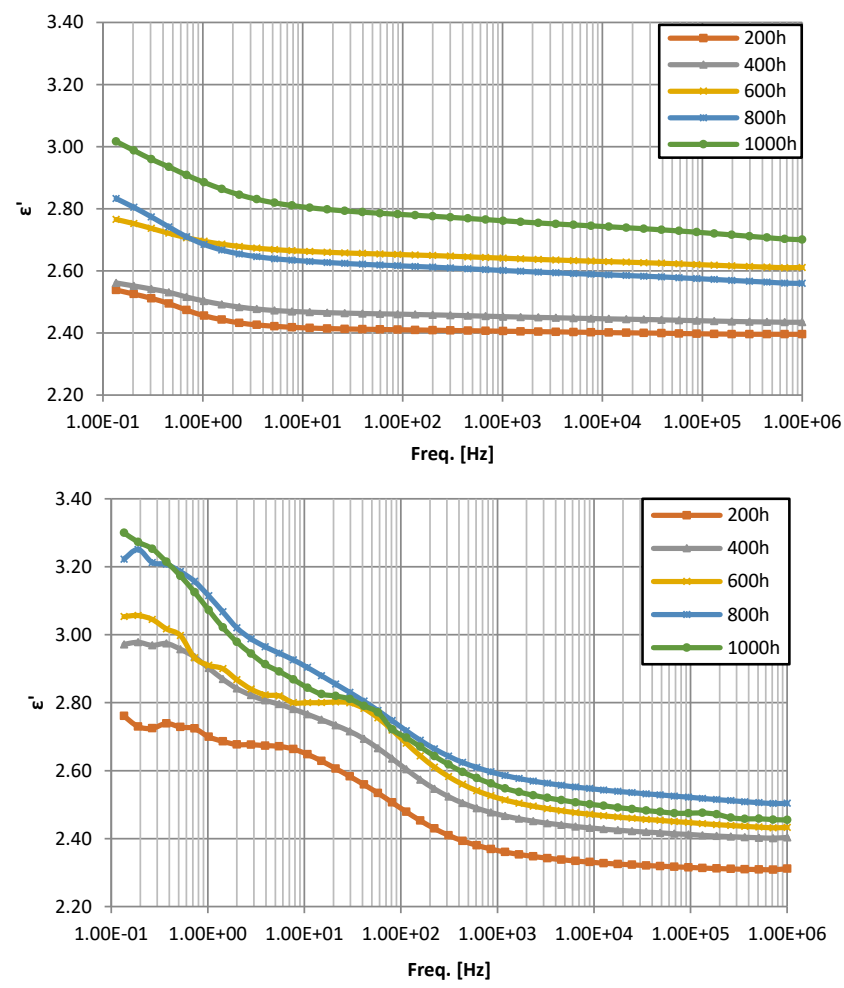

Figure 1. Dielectric spectra as a function of frequency of real part of permittivity. Test made at delivery time (a) and after four years of uncontrolled environment storage (b)

Trends of the imaginary part of permittivity at delivery time and after four years are reported in Fig. 2.a and 2.b, respectively. Imaginary permittivity has been used as a marker to qualify condition of dielectrics [6], as it is very sensitive to aging. Figures 2.a and 2.b show a completely different trend, in both high and low frequency ranges. Focusing on the 200haged sample, at high frequencies, $\varepsilon$ " increases by about three times. But the longer the aging time, the lower the raise of $\varepsilon$ ". Indeed, $\varepsilon$ " increases by only $5 \%$ in the $1000 \mathrm{~h}$-aged cable.

It is worth noting that Fig. 2.b shows a peak in the $10-100$ $\mathrm{Hz}$ range which is completely absent in the tests made at delivery time. This phenomenon can be likely imputed to additive consumption.

At low frequencies, the trend is quite unchanged. Possible little differences can be attributed to the higher-frequency peak discussed above.

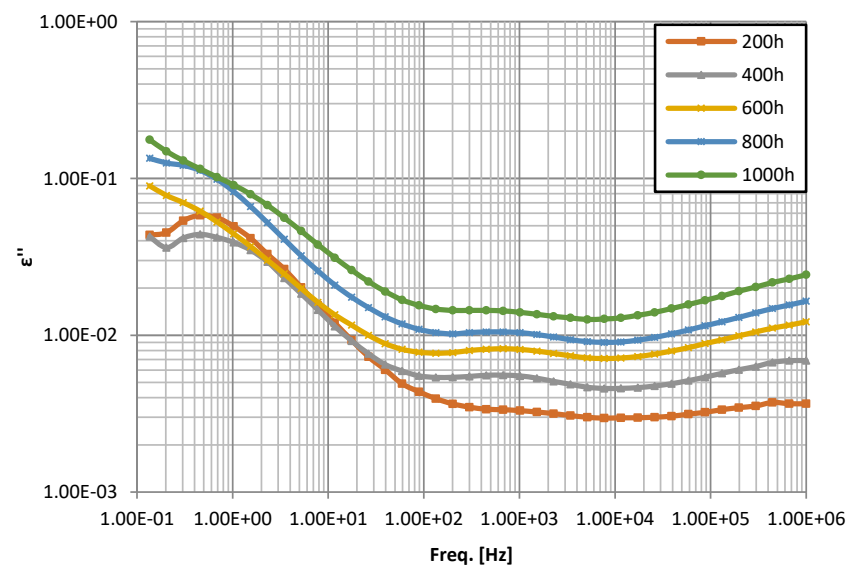




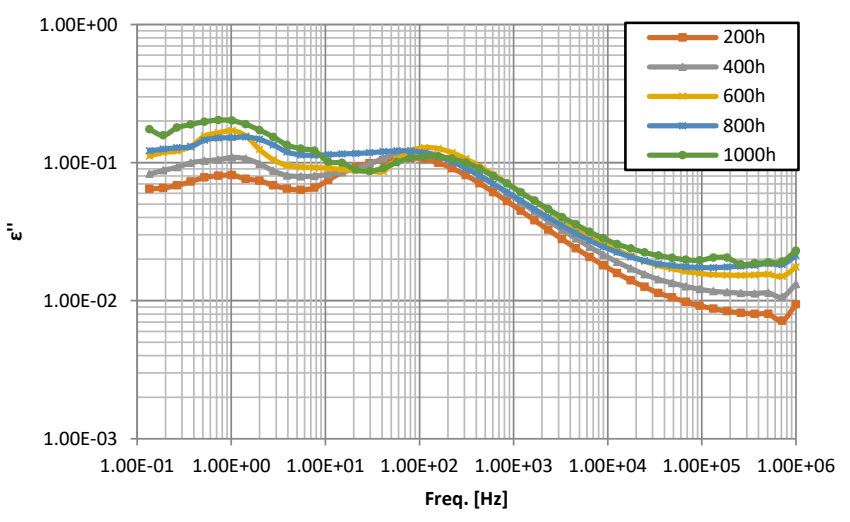

Figure 2. Imaginary part of permittivity dielectric spectra as a function of frequency. Test made at delivery time (a) and after four years of uncontrolled environment storage (b)

\subsection{Mechanical results}

Post irradiation effects may be also evidenced focusing on the mechanical tests reported here.

Elongation at break as a function of aging times is shown in Figure 3. IEC Standards [9] set insulation failure at $50 \%$ of $\mathrm{EaB}$. It can be noticed that, focusing on delivery time tests, $1000 \mathrm{~h}$ aging time has been requested in order to reach the $50 \%$ endpoint. In contrast, after four-year storage, $400 \mathrm{~h}$ of irradiation time is enough to reach the same value. This behavior may be attributed to post-irradiation effects that catalyze oxidation and crosslinking reactions even after irradiation source is turned off.

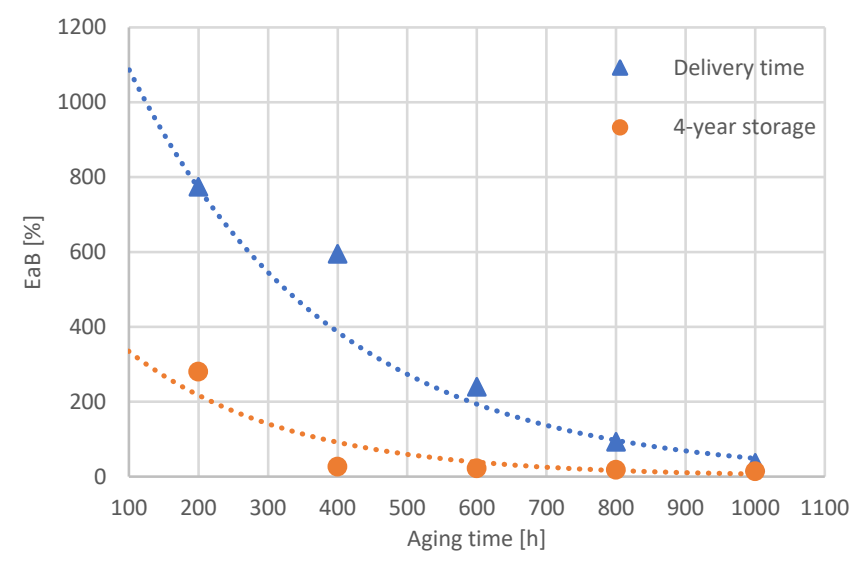

Figure 3. Elongation at break as a function of aging times. Interpolation made through an exponential trendline

\section{DISCUSSION}

As known in literature [5] oxidation, with cross-linking and chain-scission, brings to the raise of losses inside the polymeric material, which are reflected on the increase of $\varepsilon$ "' (see Fig. 2).

Post-irradiation effect can be explained through oxygen and radicals mobility inside different regions of polymers. In general, oxygen diffusion into the crystalline part of polymer is very difficult because of the difference between diffusion constants of amorphous and crystalline regions.

Radicals are generated by radiation, inside both crystalline and amorphous parts of polymers. Radicals made in the amorphous region are able to bond each other or with oxygen, while radicals made in the crystalline region remain trapped inside the crystalline matrix for some time after irradiation. These radicals slowly migrate to the interface between amorphous and crystallin regions, where they meet molecules with oxygen and start new oxidative reactions [8].

As known in literature [7], chemical reactions are limited by the time oxygen takes to diffuse into the material. Hence, forcing high dose rates, the aging treatment only affects the superficial layer of cable specimens.

The combined effect of both DLO and post-irradiation can be seen in the different behaviour dielectric spectra have at low and high frequencies, both for real and imaginary part of permittivity, as discussed above.

High frequencies $\varepsilon$ ' modifications are usually attributed to oxidation phenomena while low frequencies ones are often related to additives consumption and structural modification (such as chain scission and crosslinking) [10]. The latter causes interfacial modification between crystalline and amorphous regions of polymers, which is associated to Maxwell-Wagner-Sillars polarization [11].

In this paper a new quantity, which consider the frequency range into which different modifications of polymers occur, is introduced. This factor, called equivalent permittivity, is defined as follows:

$$
\bar{\varepsilon}_{\text {high }}=\frac{\int_{f o}^{f_{\max }} \varepsilon d f}{d f} \quad \bar{\varepsilon}_{\text {low }}=\frac{\int_{f_{\min }}^{f_{o}} \varepsilon d f}{d f}
$$

where $\varepsilon$ is the complex permittivity, $f_{0}$ the delimitation frequency considered and $f_{\min , \max }$ is the minimum and maximum frequency of analysed spectrum, respectively.

In this paper, Imaginary part of permittivity has been studied due to the already discussed properties of this parameter as an aging marker. Imaginary equivalent permittivity $\left(\bar{\varepsilon}^{\prime \prime}{ }_{\text {high }}\right.$ and $\bar{\varepsilon}^{\prime \prime}$ low $)$ for both test period and for different aging times is showed in Fig. 4. We have chosen a delimitation frequency equal to $100 \mathrm{~Hz}$. Indeed, at frequencies lower than $100 \mathrm{~Hz}$ we usually locate interfacial phenomena, while higher frequencies values are usually related to dipolar phenomena such as, e.g., oxidation.

It can be noticed that post irradiation phenomena affect both low and high frequency range of equivalent permittivity. High frequencies equivalent imaginary permittivity (Fig. 4.a) shows a linear increase with aging time imputed to oxidation. This trend can be explained through the already discussed migration phenomenon and radical production by radiation.

Focusing on the low-frequency part of equivalent imaginary permittivity, a more important increase (up to 14 times) than previous can be underlined, that can be correlated with $\mathrm{EaB}$ behavior (Fig. 3). Indeed, the dramatic decrease of EaB after storage evidences that crosslinking of radical species can occur also years after irradiation. It is worth noting that, as discussed before, a $400 \mathrm{~h}$ irradiation can reduce $\mathrm{EaB}$ from $600 \%$ (tests at delivery time) to $30 \%$ (tests after four years), while further irradiation periods do not significantly reduce the $\mathrm{EaB}$. An equivalent behavior is shown by the equivalent permittivity, thus indicating a good correlation between this factor and crosslinking grade.

Furthermore, the plot of $\bar{\varepsilon}_{\text {high }}$ and $\bar{\varepsilon}_{\text {low }}^{\prime}$ underlines small differences between the two test periods, so that the results are not reported here for the sake of brevity. 

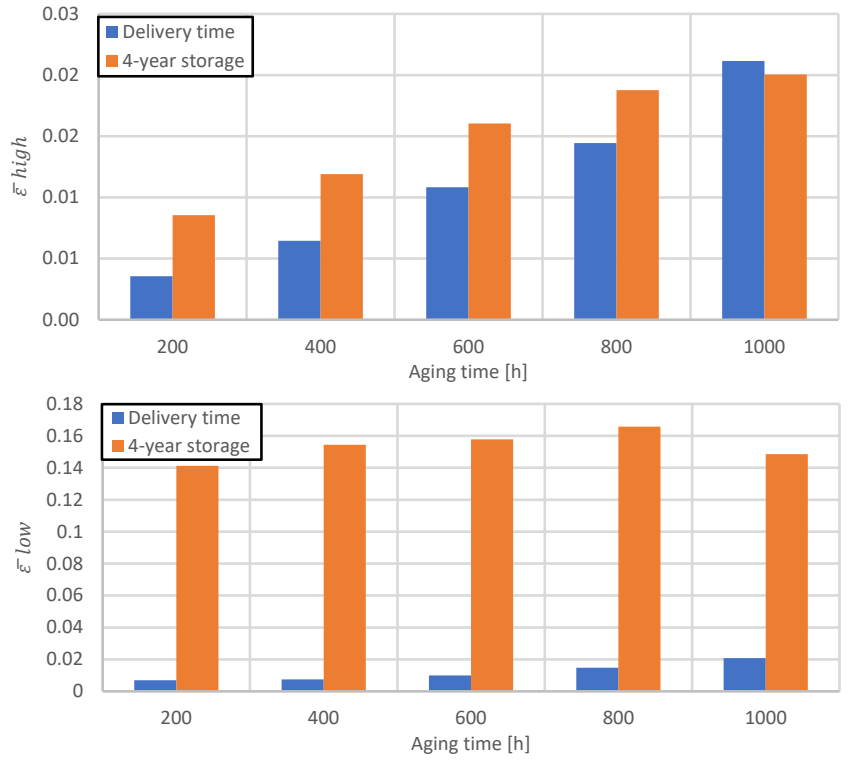

Figure 4. High (a) and low (b) frequency equivalent imaginary permittivity trend as a function of aging time for the two tests periods

\section{CONCLUSIONS}

This paper deals with electrical and mechanical properties of irradiated NPP cables which show significant changes after few years of uncontrolled environment conditions due to postirradiation effects.

Experimental tests show good correlation between dielectric and mechanical response.

Splitting dielectric spectrum into two parts we could single out different degradation effects such as oxidation, crosslinking and additives consumptions. In particular, we introduced a new factor, called equivalent permittivity, which turned out to be a good marker to quantify aging degradation of polymers, highlighting possible post irradiation effects.

Above all, during this research we were able to conclude that once a polymer is irradiated with high dose rates, like e.g. due to a nuclear accident, insulation condition changes even after few years from the event. Indeed, it has been shown that degradation evolves over time even when the radiation or other stress sources are turned off. Therefore, diagnostic measurements must be repeated and monitored even years after.

\section{ACKNOWLEDGMENT}

The research has been carried out thanks to the specimens provided within the FP7 EU project ADVANCE. The authors are grateful to Vit Placek of UJV Řež (Czech Republic) for specimen aging, Luca Verardi and Chiara Gualandi for helping with tests.

\section{REFERENCES}

[1] International Atomic Energy Agency. (2004). Management of life cycle and ageing at nuclear power plants - Improved I\&C maintenance.

[2] International Atomic Energy Agency. (2012). Assessing and managing cable aging in nuclear power plants.

[3] Laboratories S. (2005). Nuclear energy plant optimization (NEPO) - Final report on aging and condition monitoring of low-voltage cable materials.

[4] Verardi L. (2014). Aging of nuclear power plant cables: in search of non-destructive diagnostic quantities. $\mathrm{PhD}$ Thesis, University of Bologna.

[5] Verardi L, Fabiani D, Montanari GC. (2014). Correlation of electrical and mechanical properties in accelerated aging of LV nuclear power plant cables. IEEE ICHVE Poznan, Poland.

[6] Linde E, Verardi L, Fabiani D, Gedde UW. (2015). Dielectric spectroscopy as a condition monitoring technique for cable insulation based on crosslinked polyethylene. Polymer Testing.

[7] Wise J, Gillen KT, Clough RL. (1997). Quantitative model for the time development of diffusion-limited oxidation profiles. Polymer 38(8): 1929-1944.

[8] Suljovrujic E. (2013). Post-irradiation effects in polyethylenes irradiated under various atmospheres, Radiation Physics and Chemistry.

[9] IEC/IEEE 62582-3. (2012). Nuclear power plants Instrumentation and control important to safety Electrical equipment condition monitoring methods Part 3: Elongation at break.

[10] Nakamura S, Murabayashi F, Iida K, Sawa G, Ieda M. (1987). Degradation of dielectric properties of polyethylene by combined -irradiation and thermal stresses. IEEE Transactions on Electrical Insulation 22(6): 715-720.

[11] Fu M, Chen G, Dissado LA, Fothergill JC, Zou C. (2007). The effect of gamma irradiation on space charge behaviour and dielectric spectroscopy of low-density polyethylene. IEEE Conference Winchester, UK. 